\title{
Reactivity of pulmonary circulation and right ventricle function to inhaled nitric oxide in systemic sclerosis patients
}

\author{
Wojciech Plazak • Krzysztof Gryga • Jan Sznajd • \\ Joanna Wilisowska $\cdot$ Ewa Czarnobilska • \\ Grzegorz Goncerz • Piotr Podolec • Jacek Musial
}

Received: 6 November 2010 /Revised: 5 April 2011 / Accepted: 31 May 2011 /Published online: 14 June 2011

(C) The Author(s) 2011. This article is published with open access at Springerlink.com

\begin{abstract}
Systemic sclerosis ( $\mathrm{SSc}$ ) is complicated by pulmonary hypertension and right ventricle (RV) failure in approximately $10 \%$ of the patients. Factors influencing the reactivity of pulmonary circulation to vasodilators are not established, while the examination of vasoreactivity is important in determining the treatment, because systemic administration of oral vasodilators can induce severe adverse events in nonresponders. The mechanism of RV failure in SSc is unclear and may result either from increased RV afterload or intrinsic myocardial disease. The aim of the study was to assess the reactivity of pulmonary circulation to inhaled nitric oxide (iNO) and to evaluate its influence on RV function in SSc patients with elevated right ventricle systolic pressure (RVSP). In $60 \mathrm{SSc}$ patients aged 24-73 (58 females, two males; 33 patients with limited SSc and 27 with diffuse SSc), echocardiographic examination with tissue Doppler echocardiography (TDE) was performed.
\end{abstract}

W. Plazak $(\bowtie) \cdot$ J. Wilisowska $\cdot$ P. Podolec

Department of Cardiac and Vascular Diseases,

John Paul II Hospital, Jagiellonian University Medical College,

Pradnicka Str 80,

31-202 Krakow, Poland

e-mail:wplazak@szpitaljp2.krakow.pl

K. Gryga $\cdot$ J. Sznajd $\cdot$ J. Musial

Department of Internal Medicine,

Jagiellonian University Medical College,

Krakow, Poland

E. Czarnobilska

Department of Clinical and Environmental Allergology,

Jagiellonian University Medical College,

Krakow, Poland

G. Goncerz

Department of Anatomy, Jagiellonian University Medical College,

Krakow, Poland
RV function was measured by systolic (S) and early diastolic (E) velocity of tricuspid annulus by TDE. In patients with RVSP $>45 \mathrm{mmHg}$, the reactivity of pulmonary circulation was assessed by iNO test. High-resolution computerized tomography (HRCT) was performed to assess the extent of pulmonary fibrosis. Of $14 \mathrm{SSc}$ subjects with elevated RVSP (13 females, one male; RVSP 47-62 mmHg), positive reaction to iNO was observed in five (RVSP decreased from $51.6 \pm 3.7$ to $32.24 \pm 2.3 \mathrm{mmHg}$ ); nine patients were not reactive (RVSP $53.5 \pm 5.7 \mathrm{mmHg}$ before $\mathrm{iNO}$ vs. $49.6 \pm$ $6.7 \mathrm{mmHg}$ ). RV systolic function was decreased in patients with elevated RVSP as compared to the patients with normal pulmonary pressure (S velocity $13.2 \pm 1.3$ vs. $14.4 \pm 1.6 \mathrm{~cm} / \mathrm{s}$, respectively, $p<0.05$ ). Significant increase of RV systolic function during iNO test was found in reactive patients only (S velocity before iNO $12.8 \pm 1.2 \mathrm{~cm} / \mathrm{s}$, during iNO $14.5 \pm$ $1.5 \mathrm{~cm} / \mathrm{s}, p<0.01)$. RVSP decrease strongly correlated with $\mathrm{S}$ velocity increase $(r=0.95, p<0.0001)$. Response to iNO was found only in limited form of SSc; diffuse SSc patients showed no response. Pulmonary fibrosis on HRCT was more frequent in subjects nonreactive to iNO (67\% of patients) than in the reactive group ( $40 \%$ of patients). The reactivity of pulmonary circulation to iNO in $\mathrm{SSc}$ patients with elevated RVSP was found predominantly in limited form of the disease. Pulmonary fibrosis typical for diffuse SSc was more frequent in nonreactive subjects. Elevated pulmonary pressure plays an important role in RV systolic dysfunction. Pulmonary pressure decrease during iNO test leads to the improvement of RV systolic function. Therapy for right-heart failure in reactive SSc patients should be directed, if possible, at the decrease in pulmonary resistance.

Keywords Heart failure Pulmonary circulation .

Right ventricle $\cdot$ Systemic sclerosis 


\section{Objective and aim of the study}

Systemic sclerosis $(\mathrm{SSc})$ is complicated in approximately $10 \%$ of the patients by pulmonary hypertension $(\mathrm{PH})$ caused mainly by an increase in pulmonary vascular resistance [1]. The prognosis of SSc-PH without pulmonary circulation-targeted therapies is particularly poor, with estimated survival rates of approximately $50 \%$ at 2 years [1]. While prostacyclin, endothelin receptor antagonists and phosphodiestease-5 inhibitors has been shown to be partially efficacious in $\mathrm{PH}, \mathrm{SSc}-\mathrm{PH}$ remains incurable [1]. Moreover, factors influencing reactivity of pulmonary circulation to vasodilators seen in some patients [2] remain unknown [3]. Their identification may be important to make a proper choice of treatment because systemic administration of oral vasodilators can induce severe adverse events in nonresponders [4].

The second factor influencing prognosis in SSc patients is cardiac involvement. While left ventricle diastolic dysfunction plays a major role [5], recent studies based on tissue Doppler echocardiography (TDE) has also shown systolic and diastolic dysfunction involving right ventricle $[6,7]$. The mechanism of right ventricle dysfunction in SSc is not completely understood: this may involve increased right ventricle afterload caused by pulmonary hypertension as well as SSc-specific fibrotic tissue accumulation in the myocardium.

The aim of the study was to assess the reactivity of pulmonary circulation to inhaled nitric oxide (iNO) and to evaluate its influence on right ventricle function in SSc patients with elevated right ventricle systolic pressure (RVSP).

\section{Material and methods}

Study group consisted of 60 consecutive SSc patients (58 females, two males) aged 24-73 (mean age 54.4 years). The diagnosis was established according to the American College of Rheumatology standards [8]. Limited SSc was diagnosed in $33(55 \%)$ patients, and diffuse SSc in 27 (45\%). Duration of the disease was 2-32 years (mean 15.5 years). Scl-70 autoantibodies were found in $42(70 \%)$ patients, anticentromere in $16(26.7 \%)$, and other autoantibodies (anti-fibrillarin, $\mathrm{Ku}, \mathrm{Ro}$, antimitochondrial) in 13 $(21.7 \%)$. Arterial hypertension was present in four patients. Dyspnea at rest was not observed in any of the patients. The results of peripheral blood count, serum sodium, potassium, glucose, creatinine, and urinalysis were all normal. The patients were treated with $(n, \%)$ : pentoxyfilline $(42,70 \%)$, calcium blockers $(18,30 \%)$, cinnarizine $(10,16.7 \%)$, nicergoline $(7,11.7 \%)$, xantinol nicotinate $(10,16.7 \%)$, bencyclane $(7,11.7 \%)$, prednisone $(8,13.3 \%)$, angiotensin- converting enzyme inhibitors $(18,30 \%)$, diosmine (13, $21.7 \%)$, ranitidine $(8,13.3 \%)$, and hydroxizine $(13,21.7 \%)$.

In all the subjects, echocardiographic examination with tissue Doppler echocardiography was performed. The data obtained form echocardiographic examination (Toshiba Aplio SSA-770 Ultrasound System, Toshiba, Japan) included: diastolic and systolic dimension of left ventricle, ejection fraction of left ventricle (Simpson method), diastolic dimension of right ventricle, thickness of the left ventricle walls in diastole, E and A mitral inflow velocity, valvular pressures gradients, and regurgitation assessment. RVSP was calculated from the velocity of tricuspid regurgitant jet $\left(V_{\text {tr }}\right)$ according to the equation $\mathrm{RVSP}=4 V_{\mathrm{tr}}+\mathrm{RAPmmHg}$, where RAP represents right atrial pressure, estimated as 5 or $10 \mathrm{mmHg}$ due to inferior vena cava and right atrium diameters. During TDE examination, the velocities of tricuspid annulus were recorded from four-chamber view during systole ( $\mathrm{S}$ velocity) and early diastole (E velocity). Isovolumetric relaxation time (IVRT) was measured by TDE examination as the time from the end of S velocity to the onset of $\mathrm{E}$ velocity.

Based on right-heart catheterization studies, a gold standard for pulmonary artery pressure measurement, $\mathrm{RVSP}=47 \mathrm{mmHg}$ might be used as a cut-off point for $\mathrm{PH}$ screening in SSc patients by means of echocardiography [9]. In our study, in patients with RVSP exceeding $45 \mathrm{mmHg}$, the reactivity of pulmonary circulation was assessed by inhaled nitric oxide test. However it is apparent that a significant proportion of the hemodynamic response to iNO occurs at concentrations below $10 \mathrm{ppm}$ [4], we used the maximal dose of iNO (40 ppm) [4]. All the aforementioned echocardiographic and TDE parameters were determined in patients during the test (after $10 \mathrm{~min}$ of breathing NO). Positive reaction to iNO (reactive pulmonary circulation) was defined as a decrease of RVSP of more than $10 \mathrm{mmHg}$ during the test. In patients with elevated RVSP, spirometry and highresolution computerized tomography were performed to assess the extent of pulmonary involvement.

Statistical analysis was performed using Statistica Six Sigma software. All numerical data were expressed as mean values \pm standard deviations or as proportions. Continuous variables were compared by use of $t$ test. Chi-square test was used to examine differences in proportions. The relationship between the pulmonary systolic pressure change and the tricuspid annulus systolic velocity change was shown by use of linear regression with $95 \%$ confidence intervals. The level for statistical significance was predetermined at $p<0.05$.

The study was approved by the Ethical Committee. Before the study, informed consent was obtained from each patient. The study protocol conforms to the ethical guidelines of the 1975 Declaration of Helsinki. 


\section{Results}

Echocardiographic examination showed normal left ventricle dimensions $(47.7 \pm 5.9 \mathrm{~mm}$ in diastole, $30.5 \pm$ $7.5 \mathrm{~mm}$ in systole) with normal ejection fraction $(67.5 \pm$ $7.3 \%)$ in $58(96.7 \%)$ SSc patients. Left ventricle was dilated $(>56 \mathrm{~mm}$ in diastole) and ejection fraction decreased $(<55 \%)$ in two $(3.3 \%)$ patients. In contrast, mitral inflow E/A ratio was decreased $(<1.0)$ in 38 (63.3\%) patients, showing diastolic left ventricular dysfunction, that was present despite normal left ventricle muscle thickness (intraventricular septum diastolic thickness $8.9 \pm$ $1.8 \mathrm{~mm}$, posterior wall diastolic thickness $8.8 \pm 1.6 \mathrm{~mm}$ ). No signs of significant valvular disturbances were observed, excepting moderate tricuspid insufficiency in 21 (46\%) patients. Right ventricle was dilated $(>30 \mathrm{~mm}$ in parasternal view) in eight (13.3\%) patients.

Among 60 examined patients, RVSP $>45 \mathrm{mmHg}$ was found in 14 subjects, 13 females and one male. Left ventricle dimensions and function were normal in this group. RVSP values ranged from 47 to $62 \mathrm{mmHg}$ (mean $53.6 \pm 4.8 \mathrm{mmHg}$ ). In the remaining 46 patients, mean RVSP value was $28.2 \pm$ $3.7 \mathrm{mmHg}$ (ranging from 20 to $36 \mathrm{mmHg}$ ).

The dose of iNO (40 ppm) appeared safe and did not result in systemic hypotension in any of the 14 patients in which the test was performed. Out of this group, positive reaction to iNO was observed in five patients. Their mean pulmonary systolic pressures decreased from $51.6 \pm 3.7$ to $32.24 \pm 2.3 \mathrm{mmHg}$. In the remaining nine patients, pulmonary pressures did not change $(53.5 \pm 5.7 \mathrm{mmHg}$ before the test, $49.6 \pm 6.7 \mathrm{mHg}$ during the test; Fig. 1).

Right ventricle systolic function was decreased in patients with RVSP elevation as compared to the patients with normal pulmonary pressure (S velocity $13.2 \pm 1.3$ vs. $14.4 \pm$

$[\mathrm{mmHg}]$

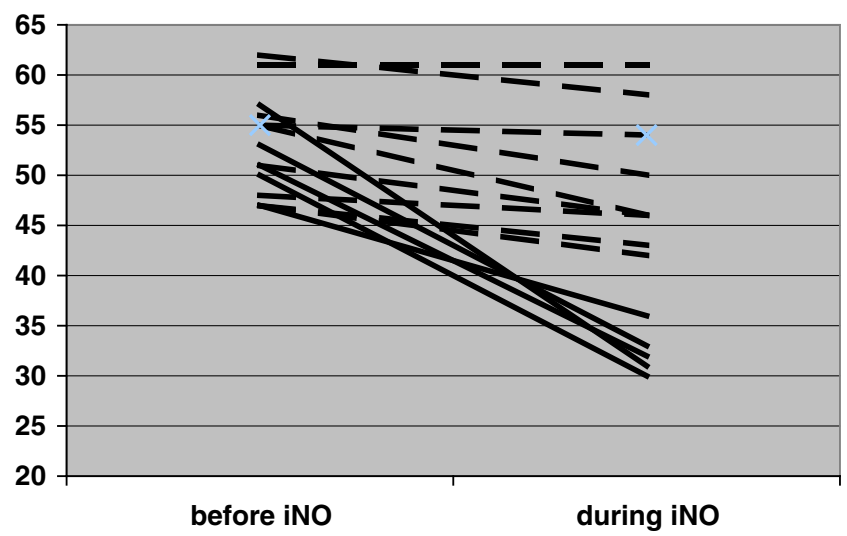

Fig. 1 Right ventricle systolic pressure in 14 patients with RVSP $>45 \mathrm{mmHg}$, before and during iNO test. Test was positive in five patients (continuous lines), in nine pulmonary circulation was not reactive to iNO (dashed lines)
$1.6 \mathrm{~cm} / \mathrm{s}$, respectively; $p<0.05$ ), diastolic right ventricle function was comparable in both groups (E velocity $12.8 \pm$ 2.2 vs. $13.4 \pm 4.2 \mathrm{~cm} / \mathrm{s}$, respectively, $p>0.05$ ). During iNO test, significant increase of right ventricle systolic function was found in the group of five patients with reactive pulmonary circulation; whereas in nonreactive patients, systolic function of right ventricle remained unchanged (Fig. 2). Diastolic right ventricle function was not changed in neither group (E velocity in reactive patients $12.9 \pm 1.2 \mathrm{~cm} / \mathrm{s}$ before iNO, $12.7 \pm 1.7 \mathrm{~cm} / \mathrm{s}$ during iNO, E velocity in nonreactive patients $12.8 \pm 3.1 \mathrm{~cm} / \mathrm{s}$ before iNO, $13.8 \pm 1.7 \mathrm{~cm} / \mathrm{s}$ during iNO).

The isovolumetric relaxation time of right ventricle (normal values, $<40 \mathrm{~ms}$ [10]) was significantly prolonged in patients with RVSP $>45 \mathrm{mmHg}$ as compared to patients with normal pulmonary systolic pressure $(90 \pm 7.7$ vs. $28 \pm$ $9.4 \mathrm{~ms}$, respectively, $p<0.001)$. In subjects with reactive pulmonary circulation, IVRT normalized from $87 \pm 5.7$ to $27 \pm 11.4$ ms during iNO test ( $p<0.001$; Fig. 3 ); whereas in nonreactive, subjects remained unchanged $(93 \pm 8.7 \mathrm{~ms}$ before iNO, $89 \pm 13.5 \mathrm{~ms}$ during iNO).

Strong correlation was found between the decrease in RVSP and the increase in tricuspid annulus systolic velocity $(r=0.95, p<0.0001$; Fig. 4$)$.

The type of the disease strongly differentiated patients with reactive from those with nonreactive pulmonary circulation. All patients with diffuse form of systemic sclerosis did not respond to iNO. On the other hand, all but one patient with limited type of the disease were reactive (Table $1 ; p<0.05$ ). Furthermore, the presence of pulmonary fibrosis on high-resolution computerized tomography (HRCT) scans was more frequent in nonreactive subjects (67\% of patients) than in the reactive group $(40 \%$ of

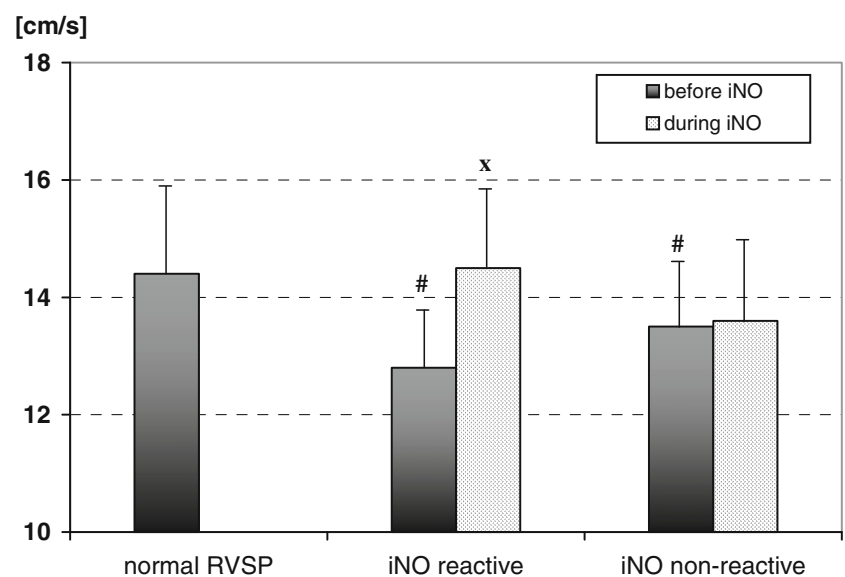

Fig. 2 Right ventricle systolic function (tricuspid systolic velocity) in patients with normal right ventricle systolic pressure (RVSP) and in patients with elevated RVSP reactive and nonreactive to iNO, ${ }^{\#} p<0.05$ according to the values in the patients with normal RVSP, ${ }^{\mathrm{x}} p<0.01$ according to the value before iNO test 


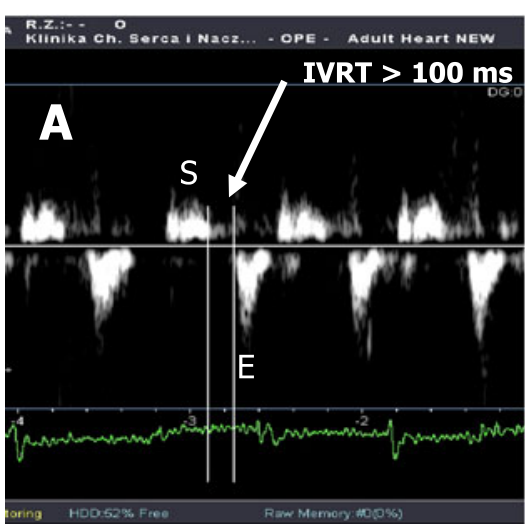

Fig. 3 Influence of iNO on right ventricle IVRT. IVRT measured by TDE represents the distance between the end of systolic $S$ wave and the beginning of early diastolic $\mathrm{E}$ wave. a In a patient with elevated

patients, $p<0.05)$. The single male in this group was not reactive. Age of the patients, duration of the disease, RVSP, and spirometry results had no influence on the reactivity of pulmonary circulation to iNO (Table 1).

\section{Discussion}

The main finding of the study includes the influence of increased right ventricle afterload due to elevation of pulmonary artery systolic pressure on right ventricle systolic dysfunction in SSc patients. Decrease of pulmonary pressure during inhaled NO test leads to right ventricle systolic function improvement.

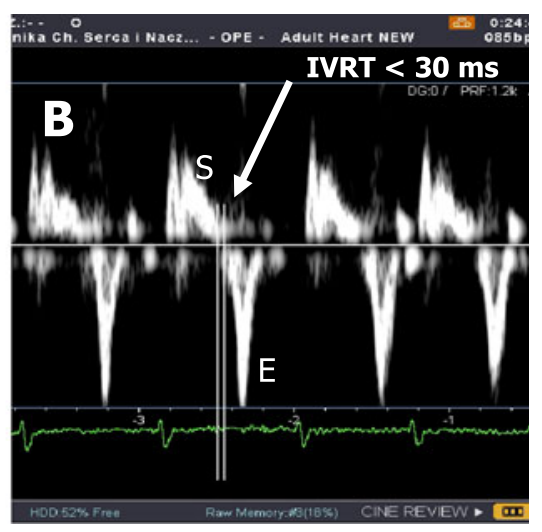

pulmonary artery systolic pressure $(57 \mathrm{mmHg})$, IVRT is prolonged $(>100 \mathrm{~ms})$. b During iNO test, pulmonary pressure decreases $(32 \mathrm{mmHg}$ ) with IVRT normalization to $<30 \mathrm{~ms}$ in this patient

Pulmonary hypertension is a devastating vascular complication of a number of connective tissue diseases, first of all systemic sclerosis, where it has a dramatic impact on the clinical course and overall survival. $\mathrm{PH}$ and pulmonary fibrosis are the most common cause of death in patients afflicted with SSc [11]. Although remarkable advances have been made to elucidate pathogenesis of idiopathic $\mathrm{PH}$ and in consequence to develop diseasetargeted therapies, the response to this therapy in SScrelated PH is suboptimal and survival remains poor [12]. While in diffuse $\mathrm{SSc}, \mathrm{PH}$ is usually secondary to interstitial lung disease, it occurs commonly also in patients with limited form of SSc [13]. Due to clinical similarity, the results of therapeutic trials in idiopathic $\mathrm{PH}$ are used to
Fig. 4 Correlation between right ventricle systolic pressure (RVSP) decrease ( $\triangle R V S P)$ and tricuspid systolic velocity increase $(\Delta S)$ during iNO test

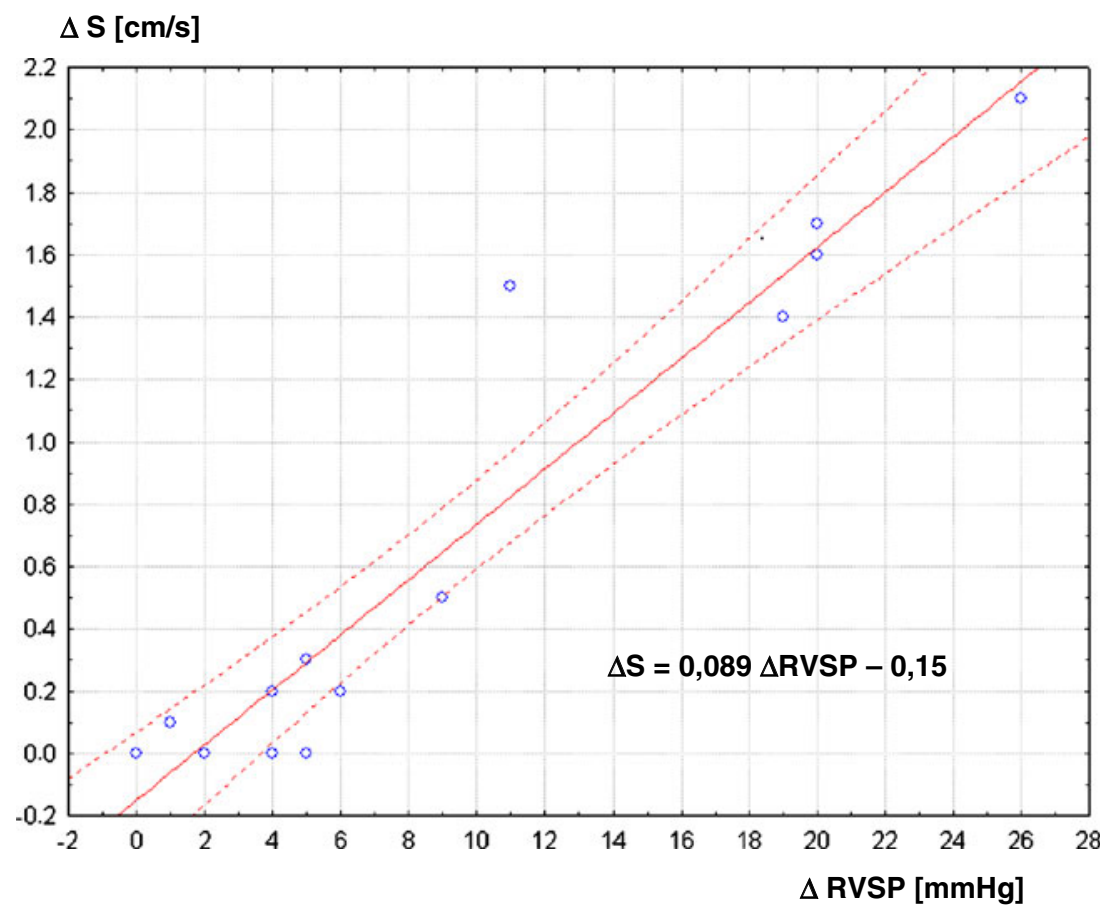


Table 1 Clinical, echocardiographic, spirometric, and tomographic data obtained in patients with pulmonary circulation reactive and nonreactive to iNO

$F V C$ forced vital capacity, FEV1 forced first-second expiratory volume, HRCT high-resolution computerized tomography

\begin{tabular}{llll}
\hline & Reactive & Nonreactive & $p$ Value \\
\hline Age (years) & $53.2 \pm 11.8$ & $57.7 \pm 3.3$ & $\mathrm{~ns}$ \\
Gender & $5(100 \%)$ females & $8(89 \%)$ females & $\mathrm{ns}$ \\
& $0(0 \%)$ males & $1(11 \%)$ male & \\
Time of the disease (years) & $4.5 \pm 1.9$ & $3.5 \pm 1.4$ & $\mathrm{~ns}$ \\
Type of the disease & $5(100 \%)$ limited & $1(11 \%)$ limited & $<0.05$ \\
& $0(0 \%)$ diffuse & $8(89 \%)$ diffuse & \\
Right ventricle systolic pressure (mmHg) & $51.6 \pm 3.7$ & $53.5 \pm 5.7$ & $\mathrm{~ns}$ \\
Spirometry-FVC (liters) & $2.71 \pm 0.9$ & $2.51 \pm 0.8$ & $\mathrm{~ns}$ \\
Spirometry-FEV1 (liters) & $2.26 \pm 0.8$ & $2.09 \pm 0.7$ & $\mathrm{~ns}$ \\
Pulmonary fibrosis in HRCT & $2(40 \%)$ present & $6(67 \%)$ present & $<0.05$ \\
& $3(60 \%)$ absent & $3(33 \%)$ absent & \\
\hline
\end{tabular}

guide treatment in $\mathrm{SSc}-\mathrm{PH}$ [12]. On the other hand, SSc patient population is becoming an important study group for the evaluation of novel pulmonary vasodilator therapies. Despite the similarities between idiopathic and SSc-related $\mathrm{PH}$, pathologic findings may vary reflecting different pathogenetic mechanisms.

Inhaled NO is a selective pulmonary vasodilator that acts preferentially on ventilated regions. Because it is rapidly inactivated by hemoglobin, this mode of administration produces little, if any, direct effect on the systemic vasculature. There is no ventilation-perfusion mismatching or hypotension, which often limit the use of conventional nonselective vasodilators [14]. In the present study, the dose of iNO (40 ppm) appeared safe and did not result in systemic hypotension in any patient.

We showed that the type of the disease and the presence of fibrosis on HRCT strongly differentiated patients with reactive from those with nonreactive pulmonary circulation. Pulmonary fibrosis in diffuse SSc patients leads to the persistent elevation of pulmonary artery systolic pressure. This condition makes the prognosis of the patients particularly grim [15]. The elevation of pulmonary artery pressure in limited SSc may result, among others, from vasospasm due to, e.g., decreased production of endogenous inducible NO synthase [16, 17]. Thus, the vasospasm and pulmonary resistance in limited SSc may react to iNO. This finding may help to select patients who may benefit from treatment with vasodilators. The therapy aimed at decreasing the pulmonary pressure is of utmost importance, not only in a long-term prognosis, but also in a short term, where even slight elevation of pulmonary resistance (pulmonary pressures in the upper normal range) leads to a significantly decreased exercise capacity [18].

Tissue Doppler echocardiography gives the possibility to assess systolic and diastolic right ventricle function that is not attainable with standard echocardiography [6]. Recent studies in SSc patients based on TDE showed both systolic and diastolic right ventricle dysfunction $[6,7]$. We showed impaired right ventricle systolic function in the patients with elevated RVSP as compared to the patients with normal pulmonary pressure. However, in patients with reactive pulmonary circulation, a significant increase in right ventricle systolic function during iNO test was demonstrated. On the contrary, in nonreactive patients, systolic function of the right ventricle remained unchanged. A drop in pulmonary systolic pressure during iNO test highly correlated with tricuspid annulus systolic velocity increase (Fig. 4).

The IVRT of the right ventricle is a sensitive marker of pulmonary pressure elevation $[10,19]$. The normalization of IVRT during iNO test confirms the decrease in pulmonary systolic pressures and improvement in RV function in reactive patients.

There are few data from literature concerning the RV function improvement during iNO test. The results based on right-heart catheterization presented by Sitbone [4] confirmed increase in right ventricle stroke volume in iNO responders. With regard to the reactivity of RV function to iNO test, we may expect the improvement of RV function during the treatment focused on the decrease in pulmonary pressure [20]. It was shown that tricuspid annulus systolic velocity above $12 \mathrm{~cm} / \mathrm{s}$ predicts the lower incidence of readmission to hospital and improves prognosis [7]. In SSc patients reactive to iNO, therapy of right-heart failure should then be focused on decreasing pulmonary resistance. The increase in tricuspid annulus systolic velocity $>12 \mathrm{~cm} / \mathrm{s}$ should be our aim while lowering pulmonary artery pressure in right-heart failure SSc patients.

\section{Limitations of the study}

Although the study was performed in $60 \mathrm{SSc}$ patients, the main findings concerning the reactivity of pulmonary circulation to iNO are based on the examination of limited group of 14 patients with elevated pulmonary artery 
systolic pressure. All measurements were performed noninvasively, without direct hemodynamic assessment. The group of patients evaluated during the study requires longterm follow up.

\section{Conclusions}

Pulmonary circulation reactive to inhaled NO was found predominantly in limited type of systemic sclerosis. The presence of pulmonary fibrosis typical for diffuse SSc was more frequent in nonreactive subjects. Increased right ventricle afterload due to elevated pulmonary artery systolic pressure is an important factor influencing right ventricle systolic dysfunction in SSc patients. Decrease of pulmonary pressure during inhaled NO test leads to right ventricle systolic function improvement. This suggests that the therapy of right-heart failure in reactive patients should be aimed if possible on the decrease in pulmonary resistance.

Acknowledgments This study was supported by a grant No N40201231/0460 from the Polish Ministry of Science and Higher Education.

\section{Disclosures None.}

Open Access This article is distributed under the terms of the Creative Commons Attribution Noncommercial License which permits any noncommercial use, distribution, and reproduction in any medium, provided the original author(s) and source are credited.

\section{References}

1. Kowal-Bielecka O, Delcroix M, Vonk-Noordegraaf A et al (2008) Outcome measures in pulmonary arterial hypertension associated with systemic sclerosis. Rheumatology (Oxford) 47(supl. 5):39-41

2. Morales-Blanhir J (2004) Clinical value of vasodilator test with inhaled nitric oxide for predicting long-term response to oral vasodilators in pulmonary hypertension. Respir Med 98:225-234

3. Sasahara A (2009) Pulmonary arterial hypertension in systemic sclerosis: can we predict responders for successful therapy? (editorial). J Rheumatol 36:665-666

4. Sitbon O, Brenot F, Denjean A et al (1995) Inhaled nitric oxide as a screening vasodilator agent in primary pulmonary hypertension: a dose-response study and comparison with prostacyclin. Am J Respir Crit Care Mad 151:384-389
5. Plazak W, Zabinska-Plazak E, Wojas-Pelc A et al (2002) Heart structure and function in systemic sclerosis. Eur J Dermatol $12: 257-262$

6. D'Andrea A, Stisi S, Bellissimo S et al (2005) Early impairment of myocardial function in systemic sclerosis: non-invasivasive assessment by Doppler myocardial and strain rate imaging. Eur $\mathbf{J}$ Echocardiog 6:407-418

7. Hsiao SH, Lee CY, Chang SM et al (2006) Right heart function in scleroderma: insight from myocardial Doppler tissue imaging. J Am Soc Echocardiogr 19:507-514

8. Smolen J, Weisman M (2008) Connective tissue disorders. In: Hochberg M, Silman A, Smolen J, Weinblatt M, Weisman M (eds) Rheumatology. Mosby Elsevier, Philadelphia, pp 1205-1485

9. Hsu VM, Moreyra AE, Wilson AC et al (2008) Assessment of pulmonary arterial hypertension in patients with systemic sclerosis: comparison of noninvasive tests with results of right-heart catheterization. J Rheumatol 35:458-465

10. Lindqvist $P$, Waldenstrom A, Wikstrom G et al (2006) Right ventricular myocardial isovolumic relaxation time and pulmonary pressure. Clin Physiol Funct Imaging 26:1-8

11. Le Pavec J, Humbert M, Mouthon L et al. (201) Systemic sclerosis-associated pulmonary arterial hypertension. Am J Respir Crit Care Med (in press).

12. Kowal-Bielecka O, Landewe R, Avouac J et al (2009) EULAR recommendations for the treatment of systemic sclerosis: a report from the EULAR Scleroderma Trials and Research group (EUSTAR). Ann Rheum Dis 68:620-628

13. Breit SN, Thornton SC, Penny R (1994) Lung involvement in scleroderma. Rev Clin Dermatol 12:243-252

14. Williamson DJ, Hayward C, Rogers P et al (1996) Acute hemodynamic responses to inhaled nitric oxide in patients with limited scleroderma and isolated pulmonary hypertension. Circulation 94:477-482

15. Mathai SC, Hummers LK, Champion HC et al (2009) Survival in pulmonary hypertension associated with the sclerodrma spectrum of diseases: impact of interstitial lung disease. Arthritis Rheum 60:569-577

16. Allanore Y, Borderie D, Hilliquin P et al (2001) Low levels of nitric oxide $(\mathrm{NO})$ in systemic sclerosis: inducible NO synthase production is decreased in cultured peripheral blood monocyte/ macrophage cells. Rheumatology (Oxford) 40:1089-1096

17. Menkes CJ, Allanore Y, Borderie D et al (2001) Inducible nitric oxide synthase expression and nitric oxide production by monocytes in systemic sclerosis. Bull Acad Natl Med 185:509-522

18. Kovacs G, Maier R, Aberer E et al (2009) Borderline pulmonary arterial pressure is associated with decreased exercise capacity in scleroderma. Am J Respir Crit Care Med 180:881-886

19. Brechot N, Gambotti L, Lafitte S et al (2008) Usefulness of right ventricular isovolumic relaxation time in predicting systolic pulmonary artery pressure. Eur J Echocardiogr 9:547-554

20. Freyhaus H, Dumitrescu D, Bovenschulte H et al (2009) Significant improvement of right ventricular function by imatinib mesylate in scleroderma-associated pulmonary arterial hypertension. Clin Res Cardiol 98:265-267 